\title{
Anatomía radiológica de la base de cráneo y los nervios craneales parte 2: Nervios craneales
}

\author{
Andrés Miranda-Merchak ${ }^{1,2 *}$, Cristina Kuschel R. ${ }^{2,3}$, Maximiliano Miranda G. ${ }^{2,4}$, Aníbal Fuentes G. ${ }^{2,4}$ \\ 1. Neurorradiólogo, Servicio Neurorradiología, Hospital Barros Luco Trudeau. Santiago, Chile. \\ 2. Servicio Radiología, Clínica Dávila. Santiago, Chile. \\ 3. Residente Radiología, Universidad de Los Andes Santiago, Chile. \\ 4. Radiólogo, Universidad Mayor. Santiago, Chile.
}

\section{Skull base and cranial nerves radiologic anatomy part 2: Cranial nerves}

\section{Resumen:}

La anatomía de la base del cráneo es compleja. Numerosas estructuras neurovasculares vitales pasan a través de múltiples canales y agujeros ubicados en la base del cráneo. Con el avance de la tomografía computarizada (TC) y la resonancia magnética (RM), es posible la localización cada vez más precisa de lesiones y la evaluación de su relación con las estructuras neurovasculares adyacentes. El trayecto de los nervios craneales sigue un recorrido conocido y se transmiten a la cara y cuello por los forámenes de base de cráneo. La tomografía computada y la resonancia magnética son complementarias entre sí y, a menudo, se usan juntas para demostrar la extensión total de la enfermedad. La segunda parte de esta revisión se centra en el estudio radiológico de los nervios craneales.

Palabras clave: Base de cráneo; Nervios craneales; Resonancia magnética; Tomografía computada.

\begin{abstract}
:
The skull base anatomy is complex. Many vital neurovascular structures course through the skull base canals and foramina. With the advancement of CT and MRI, the localization of lesions has become more precise as their relationship with adjacent neurovascular structures. There is a known course of the cranial nerves as well as their skull base exiting foramina to the head and neck. CT and MRI are complimentary modalities and are often used together to map the full extent of disease. The second article in this review focus on the radiologic study of the cranial nerves.
\end{abstract}

Keyword: Cranial nerves; CT; MRI; Skull base.

Miranda A., et al. Anatomía radiológica de la base de cráneo y los nervios craneales parte 2: Nervios craneales. Rev Chil Radiol 2020; 26(2): 62-71.

*Correo electrónico: Andrés Miranda / andresrobertomiranda@gmail.com

Trabajo enviado el 28 de enero de 2020. Aceptado para publicación el 04 de mayo de 2020.

\section{Introducción}

La anatomía de los nervios craneales es compleja ${ }^{(1)}$. Numerosas estructuras neurovasculares vitales pasan a través de múltiples canales y agujeros ubicados en la base del cráneo, conectando estructuras intracraneanas con el oído, las cavidades nasales, los senos paranasales, órbitas y cuello suprahioideo(2). Es necesario tener una rápida y actualizada aproximación a la anatomía de la base de cráneo, y conocer qué técnica de imagen se debe utilizar para poder optimizar su análisis y evaluar los distintos procesos patológicos que pueden afectarla. Existen doce pares de nervios craneales numerados en su orden de emergencia desde craneal a caudal ${ }^{(3,4)}$.

La RM es la técnica de elección para evaluar los nervios craneales. No obstante, los forámenes de 
la base de cráneo son mejor visualizados en $\mathrm{TC}^{(5)}$. El objetivo de este artículo es graficar de manera simplificada la anatomía en TC y RM de los nervios craneales ${ }^{(3)}$.

\section{Anatomía y nervios craneales}

A excepción del nervio olfatorio (I) y nervio óptico (II) que son haces de sustancia blanca con conexión a cortezas primarias (ver la discusión más abajo), los nervios craneales poseen núcleo en el parénquima cerebral. Luego de emerger o ingresar al troncoencéfalo, cada nervio craneal cursa a través del líquido cerebro raquídeo (LCR), luego atraviesa las paquimeninges, seguido de un compartimento venoso (a excepción del VII y VIII), extendiéndose a través de agujeros de la base del cráneo para ingresar a la cara y cuello. Durante este trayecto, los nervios están cubiertos por LCR en el espacio subaracnoideo, sangre venosa en el espacio interdural, hueso al atravesar los forámenes de la base de cráneo y partes blandas al salir del cráneo. Además, el trayecto anatómico de cada nervio craneal es distinto, algunas consideraciones anatómicas fundamentales son suficientemente similares para permitir una clasificación sistemática de los diferentes segmentos, compartiendo propiedades imagenológicas similares (Figura 1) ${ }^{(6)}$.

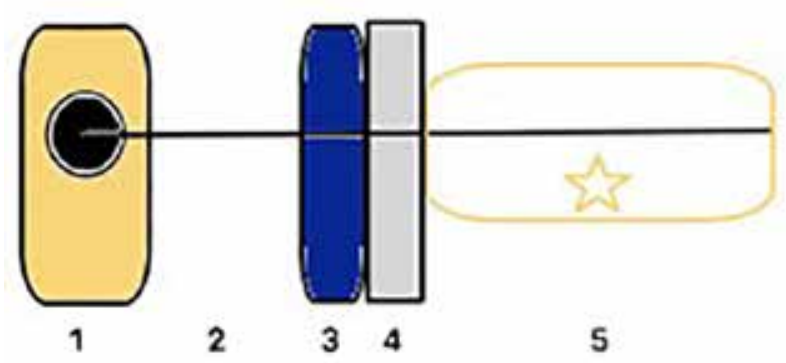

Figura 1: Esquema representativo del origen en el tronco encefálico (1), trayecto cisternal (2), senos venosos (3), foramen óseo (4) hasta su órgano blanco (5).

Los nervios craneales poseen recorridos complejos, por lo que es necesario conocer su trayecto y los forámenes de la base de cráneo que atraviesan, los que se detallaran a continuación.

\section{Nervio olfatorio (NC I)}

Es el primer y más rostral de los nervios craneales.

Los segmentos del nervio olfatorio son: Epitelio nasal, segmento transetmoidal, bulbo olfatorio intracraneal, tracto olfatorio estría medial y lateral, y corteza olfatoria $^{(7)}$.
La célula bipolar es la neurona sensorial ubicada en el techo de la cavidad nasal, inmediatamente inferior a la lámina cribosa del hueso etmoides. Sus procesos centrales forman filamentos (aproximadamente 20) que pasan a través de la placa cribosa, perforan la duramadre y se retransmiten al bulbo olfatorio, la porción anterior expandida del nervio que se encuentra en la fosa olfatoria. Estas neuronas forman el nervio olfatorio que pasa posteriormente dentro del surco olfatorio de la fosa craneal anterior y luego entre el giro recto y el giro orbitario medial. Anterior a la sustancia perforada anterior, forman las estrías olfativas medial y lateral. Las estrías olfatorias laterales se proyectan hacia el uncus. Las estrías olfatorias mediales finalmente se proyectan hacia los núcleos del hipotálamo y el tronco encefálico.

Las imágenes coronales son las mejores para representar el nervio olfatorio, que está situado en las profundidades del surco olfatorio (Figura 2).
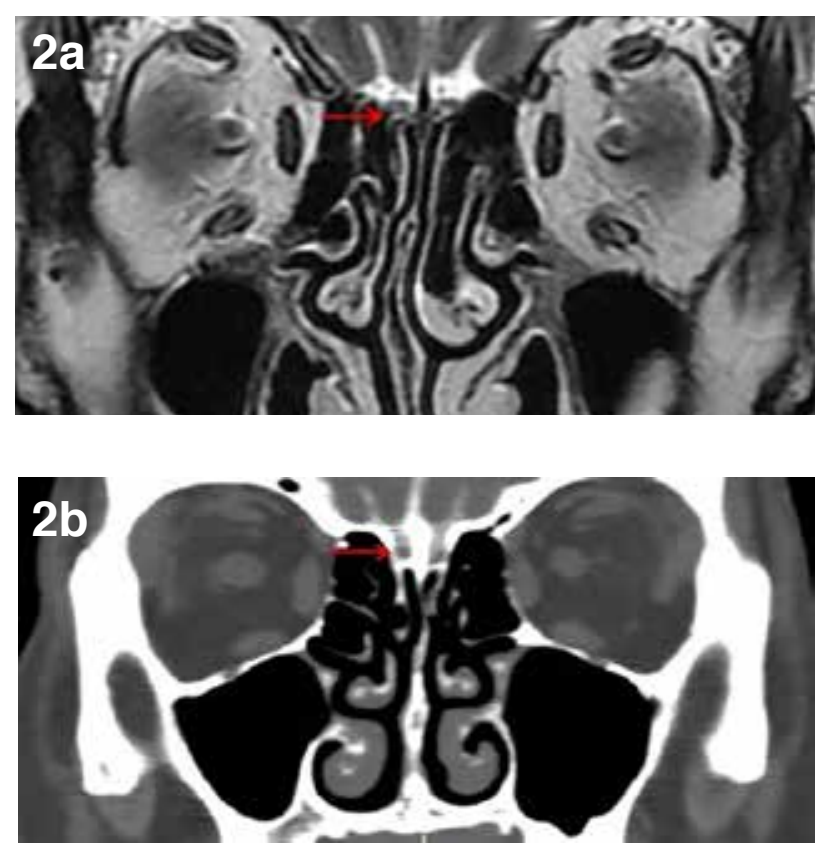

Figura 2: Corte coronal de RM potenciada en T2 (a) y de cisterno TC (b) del bulbo olfatorio derecho. (Flecha roja).

\section{Nervio óptico (NC II)}

Junto con el nervio olfatorio constituyen una extensión del sistema nervioso central. Sus procesos centrales hacen sinapsis sobre las células ganglionares en la superficie vítrea de la retina y pasan a través del disco óptico fuera del globo y forman el nervio óptico propiamente tal.

Los segmentos del nervio óptico son: segmento intraocular, segmento intraorbitario, segmento intracanalicular, segmento intracraneal, quiasma óptico, tracto óptico, radiación óptica y corteza visual(7). 
- Segmento intraocular: se encuentra dentro de la retina y emerge a través de una abertura escleral ${ }^{(7)}$.

- Segmento intraorbitario: pasa hacia posterior y centralmente dentro de la órbita y está rodeado por revestimiento dural y LCR; por lo tanto, se comunica directamente con el espacio subaracnoideo (Figura 3).

- Segmento intracanalicular: donde el nervio óptico emerge, a través, del anillo tendinoso y el canal óptico superior a la arteria oftálmica (Figura 4).

- Segmento intracraneal o cisternal: ingresa a la fosa craneal media y pasa dentro de la cisterna quiasmática en la región supraselar con la arteria cerebral anterior en su parte superolateral, uniéndose con el nervio óptico contralateral en el quiasma óptico (Figura 4).
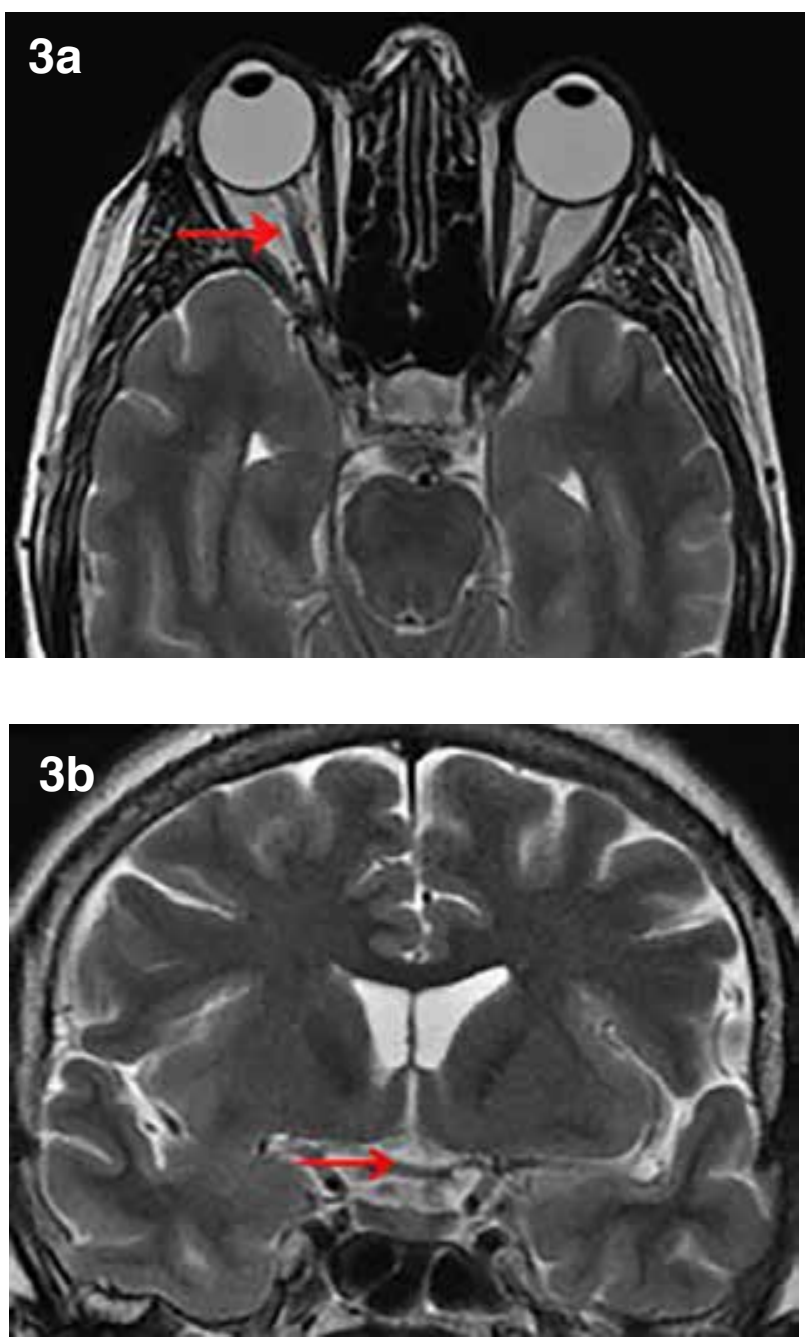

Figura 3: Corte axial (a) y coronal (b) en RM potenciada en T2, muestra el trayecto de ambos nervios ópticos y quiasma óptico respectivamente.

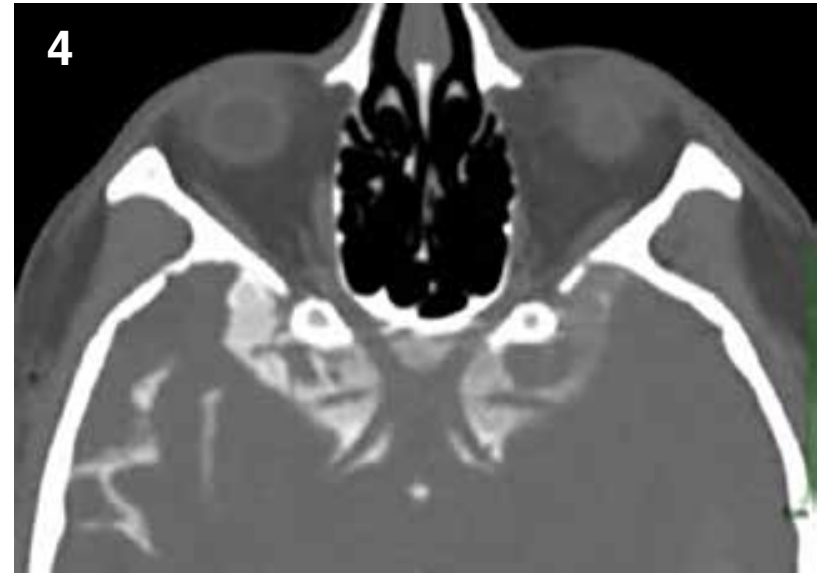

Figura 4: Corte axial de cisterno TC con ventana de partes blandas, se observa ambos tractos, quiasma y nervios ópticos en su trayecto orbitario, canalicular y cisternal.

- Quiasma óptico: las fibras nasales de cada nervio óptico se decusan mientras que las fibras temporales no (decusación parcial). Del quiasma óptico surgen dos vías ópticas, cada una de las cuales contiene fibras nasales del nervio óptico contralateral y fibras temporales del nervio óptico ipsilateral (Figura 3 y 4).

- Tracto óptico: El tracto óptico recorre el pedúnculo cerebral para transmitirse en el cuerpo geniculado lateral del tálamo.

- Radiaciones ópticas: Surgiendo del cuerpo geniculado lateral del tálamo, las radiaciones ópticas se dividen en haces superiores e inferiores. El haz superior transporta información del cuadrante superior de la retina que representa el campo visual inferior y termina en el aspecto superior del surco calcarino (cuña). El haz inferior (asa de Meyer) transporta información desde el cuadrante inferior de la retina que representa el campo visual superior que se bifurca en sentido anterior en el lóbulo temporal y forma la pared lateral de la asta temporal del ventrículo lateral y luego pasa hacia el extremo inferior del surco calcarino (giro lingual).

\section{Nervio oculomotor (NC III)}

Es responsable de los movimientos de cuatro de los seis músculos extraoculares, los otros dos están inervados por los nervios troclear y abducens. Surge del mesencéfalo anterior ${ }^{(7)}$.

- Segmento nuclear y parenquimatoso: El núcleo oculomotor se encuentra en la materia gris periacueductal profunda a nivel del colículo superior anterior al acueducto cerebral. Las fibras atraviesan el tegmento, el núcleo rojo y la cara medial de la sustancia negra para emerger por el lado medial del pedúnculo cerebral hacia la cisterna interpeduncular ${ }^{(7)}$. 
- Segmento cisternal: El nervio luego viaja hacia anterior, por inferior de la arteria cerebral posterior (donde puede ser comprimido por un aneurisma de la arteria comunicante posterior) y por superior de la arteria cerebelosa superior, antes de perforar la duramadre y entrar al seno cavernoso (Figura 5).

- Segmento cavernoso: Dentro del seno cavernoso, el nervio oculomotor se localiza en la parte superior de la pared lateral, por sobre el nervio troclear (Figura 6).

- Segmento extraforaminal: Entra en la órbita a través de la fisura orbitaria superior como dos ramas: división superior y división inferior, con el nervio nasociliar (rama de la división oftálmica del nervio trigémino) entre ellos y el nervio abducens (CN VI) debajo de los tres. Estas
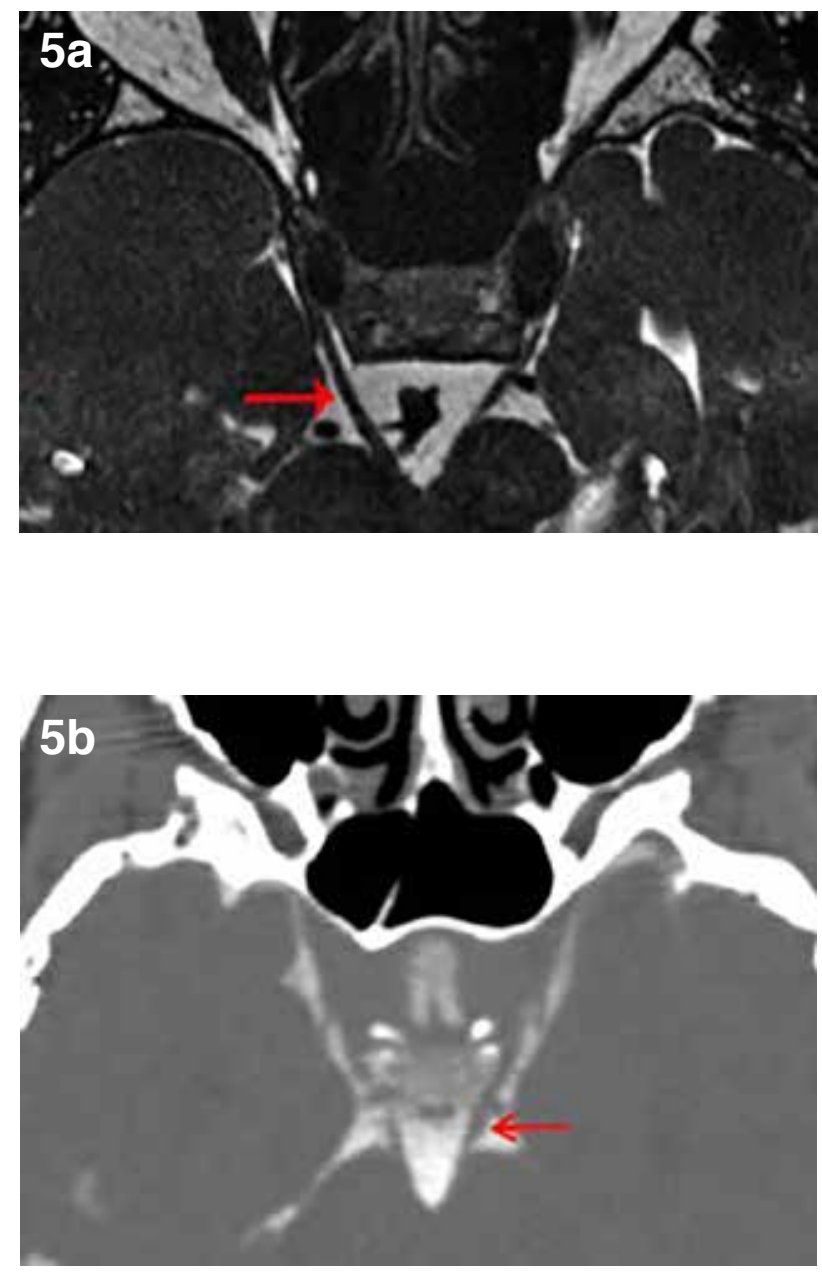

Figura 5: Corte axial a nivel de la emergencia de ambos nervios oculomotores en la cisterna interpeduncular en RM en secuencia FIESTA y cisterno TC. cuatro ramas pasan por el anillo tendinoso de Zinn, creado por la inserción de los músculos extraoculares. La división superior pasa por superior al nervio óptico y da ramas motoras al recto superior y al músculo elevador del párpado superior. Además, inerva a este último con fibras simpáticas derivadas de la arteria carótida interna. La división inferior inerva el recto inferior, el recto interno (transcurriendo por inferior al nervio óptico) y el oblicuo inferior. También emite la raíz parasimpática al ganglio ciliar $^{(7)}$.

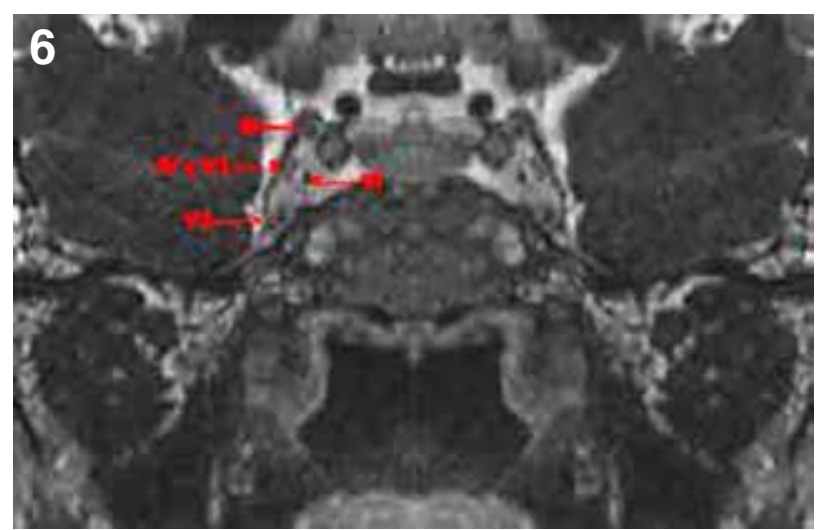

Figura 6: Corte coronal de RM secuencia FIESTA con gadolinio, centrada en seno cavernoso demuestra a izquierda, de craneal a caudal NC III, IV, V1, IV y V2. A derecha su representación en espejo sin indicadores.

\section{Nervio troclear (NC IV)}

Es el nervio motor del músculo oblicuo superior del ojo. Es de difícil visualización por su pequeño tamaño. Se puede dividir en cuatro partes:

- Segmento nuclear y parenquimatoso: El núcleo troclear se encuentra en el mesencéfalo dorsal, anterior a la sustancia gris periacueductal. Sus fibras se dirigen dorsalmente y decusan dorsalmente a la sustancia gris periacueductal antes de emerger del tronco encefálico inmediatamente por inferior del colículo inferior. Es el único nervio craneal que emerge del tronco encefálico posteriormente ${ }^{(7)}$.

- Segmento cisternal: El nervio rodea los pedúnculos cerebrales en la cisterna. Junto con el nervio oculomotor (NC III), se extiende entre la arteria cerebelosa superior y la arteria cerebral posterior y perfora la duramadre entre el borde libre y el adjunto a la tienda del cerebelo. Es el nervio craneal de mayor longitud del trayecto cisternal (Figura 7). 


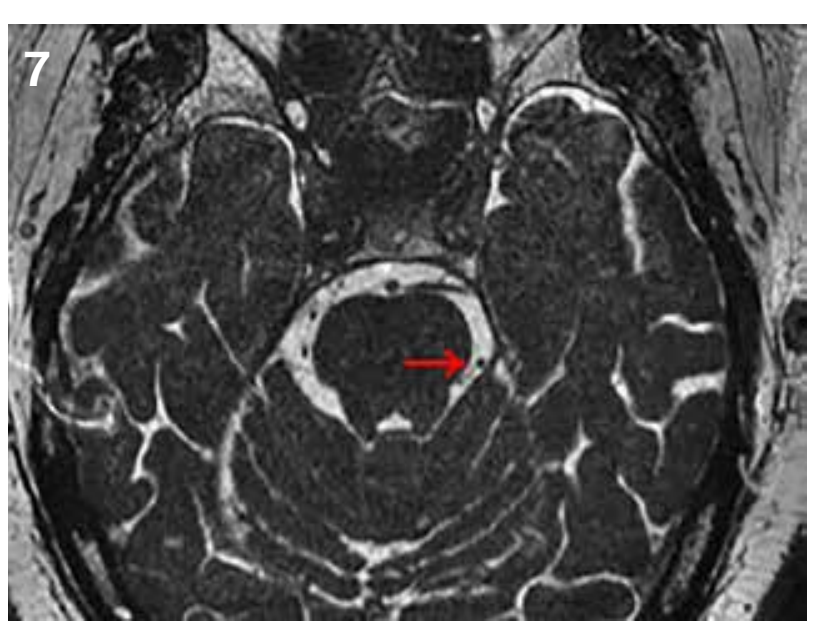

Figura 7: Nervio troclear (IV par), apenas visible en secuencia FIESTA con voxeles de 0,6 $\mathrm{mm}$.

- Segmento del seno cavernoso: Dentro del seno cavernoso, el nervio troclear se localiza inicialmente por inferior del nervio oculomotor en la pared lateral del seno, aunque para cuando alcanza la fisura orbitaria superior, se encuentra por superior (fuera del anillo tendinoso) (Figura 6).

- Segmento extraforaminal: Entra en la órbita exterior y es superolateral al anillo tendinoso, entre la vena oftálmica superior y el cuadrante superolateral del anillo. Una vez en la órbita, se arquea hacia superior y medial sobre el recto superior y el elevador del párpado superior para inervar el oblicuo superior ${ }^{(7)}$.

\section{Nervio trigémino (NC V)}

Su función principal es transmitir información sensorial de la cara y la cabeza, y proporciona control motor a los músculos de la masticación. Tiene múltiples núcleos en el tronco cerebral (sensorial y motor) así como múltiples interconexiones con otros nervios craneales. Intercambia las fibras parasimpáticas y las fibras del gusto, y se divide en numerosas ramas terminales ${ }^{(7)}$.

- Segmento nuclear: Hay tres núcleos sensoriales y uno motor. Los núcleos sensoriales están dispuestos en una columna que se extiende desde el mesencéfalo a través de la protuberancia y el bulbo y hacia la médula cervical superior.

- Núcleo mesencefálico: fibras propioceptivas para los músculos de la cara, la órbita, la masticación y la lengua.

- Núcleo sensorial principal: ubicado en la parte superior de la protuberancia, lateral al núcleo del motor es responsable de la sensación táctil de las tres divisiones del trigémino.

- Núcleo espinal: la protuberancia inferior al cordón cervical superior es responsable del dolor y la temperatura; además, recibe fibras aferentes del nervio glosofaríngeo y del nervio vago ${ }^{(7)}$.

- Núcleo motor: se encuentra en la parte superior de la protuberancia y emite la raíz motora más pequeña que deriva del ganglio trigeminal e inerva los músculos de la masticación, así como también miohioideo y tensor del velo palatino(7).

- Segmento cisternal y dural: El nervio trigémino emerge en la parte media de la protuberancia anterior, pasa a través de la cisterna prepontina (porción cisternal) para ingresar al cavum de Meckel donde sus fibras forman el ganglio trigeminal, que también se conoce como el ganglio de Gasser o semilunar (Figuras 8 y 9 ).
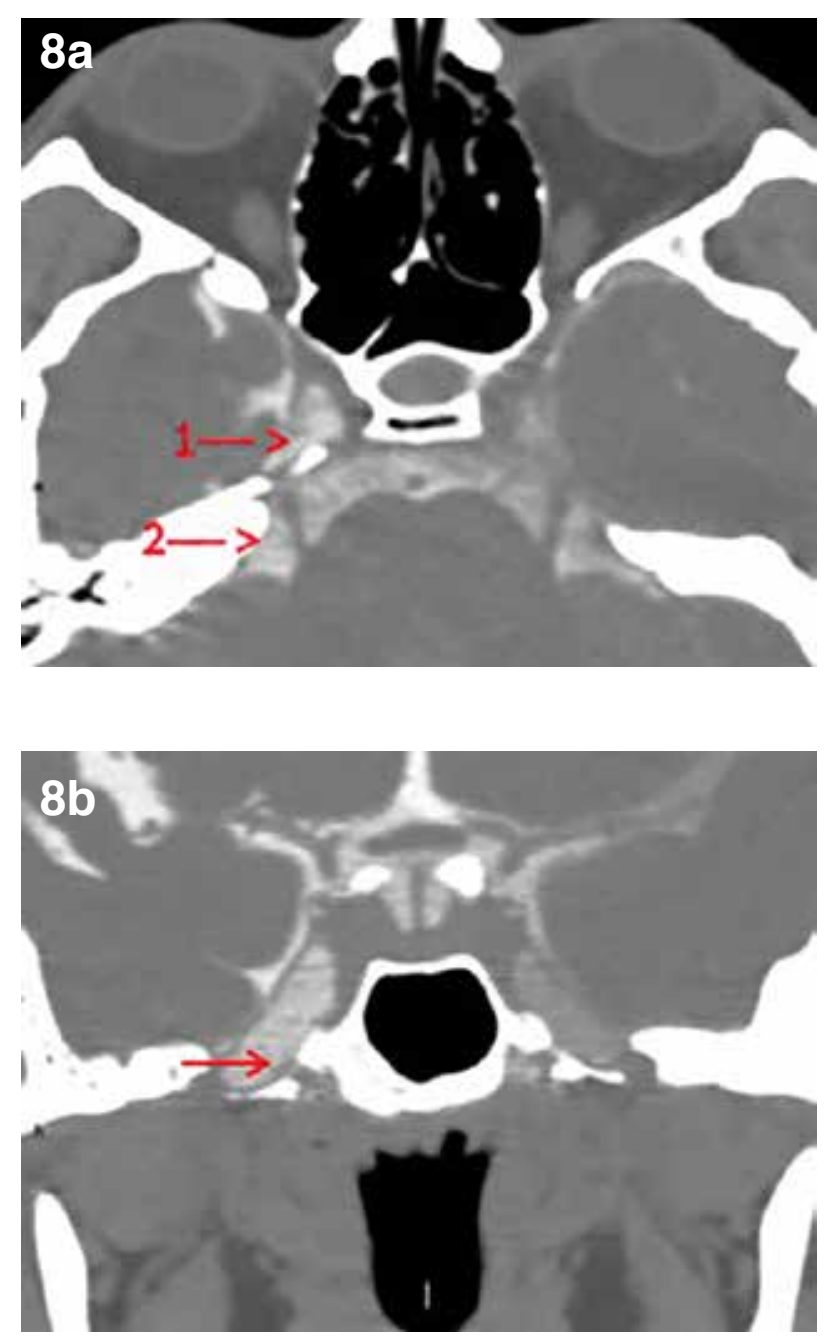

Figura 8: Corte axial y coronal de cisterno TC que demuestra el cavum de Meckel (a.1 y b) y nervio trigémino (a.2). 

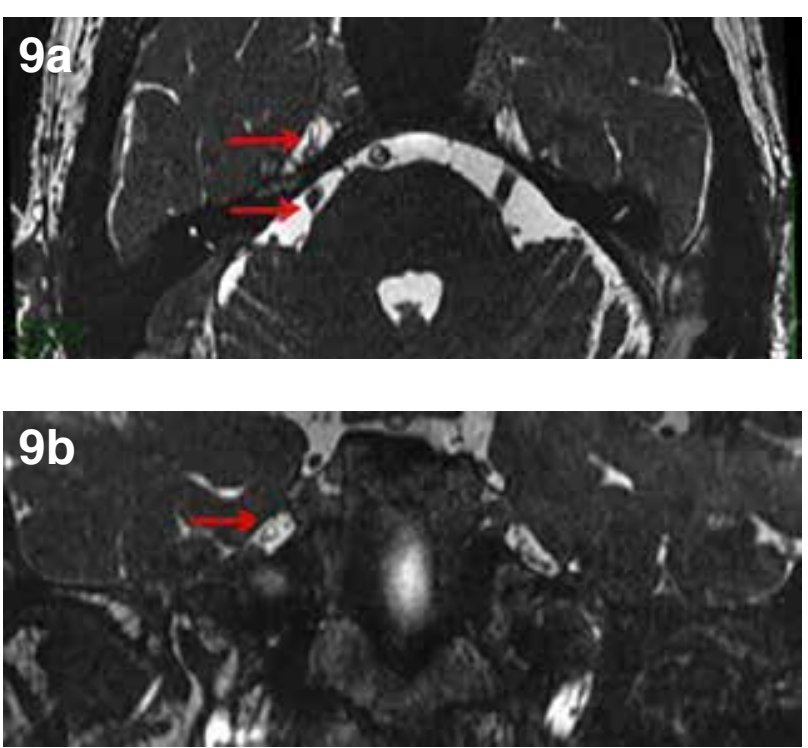

Figura 9: Corte axial (a) y coronal (b) de secuencia FIESTA demostrando (a) el trayecto cisternal y cavum de Meckel del V par craneal. (b) Cavum de Meckel.

Luego se divide en tres ramas principales:

- Segmento del seno cavernoso y extraforaminal, Nervio oftálmico (V1): cursa en la pared lateral del seno cavernoso inferior al nervio troclear. Justo antes de entrar en la órbita, surge el nervio tentorial y asciende para suministrar una gran parte de la hoz y la duramadre supratentorial. La división oftálmica luego se divide en 3 ramas terminales antes de que cada una transcurra a través de la fisura orbital superior por separado ${ }^{(7)}$.

- Segmento del seno cavernoso y extraforaminal, Nervio maxilar (V2): Cursa anteriormente en la pared lateral del seno cavernoso inferior al V1. Justo antes de salir del cráneo transcurre a lo largo del piso de la fosa craneal media y emite el nervio meníngeo medio que asciende para inervar la duramadre anterior de la fosa craneal media. Luego pasa a través del foramen redondo en el ala mayor del hueso esfenoides para emerger del cráneo e ingresar a la fosa pterigopalatina. Da ramas al ganglio pterigopalatino y recibe nervios parasimpáticos del ganglio a través del nervio petroso mayor ${ }^{(7)}$.

- Segmento extraforaminal, Nervio mandibular (V3): Cursa inferiormente a través del foramen oval para entrar en la fosa infratemporal, por lo tanto, no pasa a través del seno cavernoso. Consiste en una raíz sensorial y una raíz motora más pequeña, la última que deriva del ganglio trigeminal. Estas raíces pasan a través del foramen oval por separado y luego se unen justo por inferior al foramen ${ }^{(7)}$.
Nervio abducens (NC VI)

Inerva el músculo recto lateral y puede dividirse en cuatro segmentos:

- Segmento nuclear: El núcleo abducens es un pequeño núcleo situado en la parte superior de la fosa romboidal por inferior al colículo del facial dentro de la protuberancia ${ }^{(7)}$.

- Segmento cisternal y dural: Es el nervio más medial que emerge inmediatamente por inferior de la protuberancia en la unión pontomedular en la cisterna prepontina. Se dirige hacia el clivus en dirección superior y lateral a lo largo del clivus encerrado dentro de una vaina fibrosa llamada canal de Dorello y perfora la duramadre inferior al proceso clinoideo posterior. A continuación, recorre el ápex del peñasco medial hacia el seno cavernoso, teniendo un trayecto oblicuo y una posición relativamente anclada en el canal de Dorello (Figuras 10 y 11).
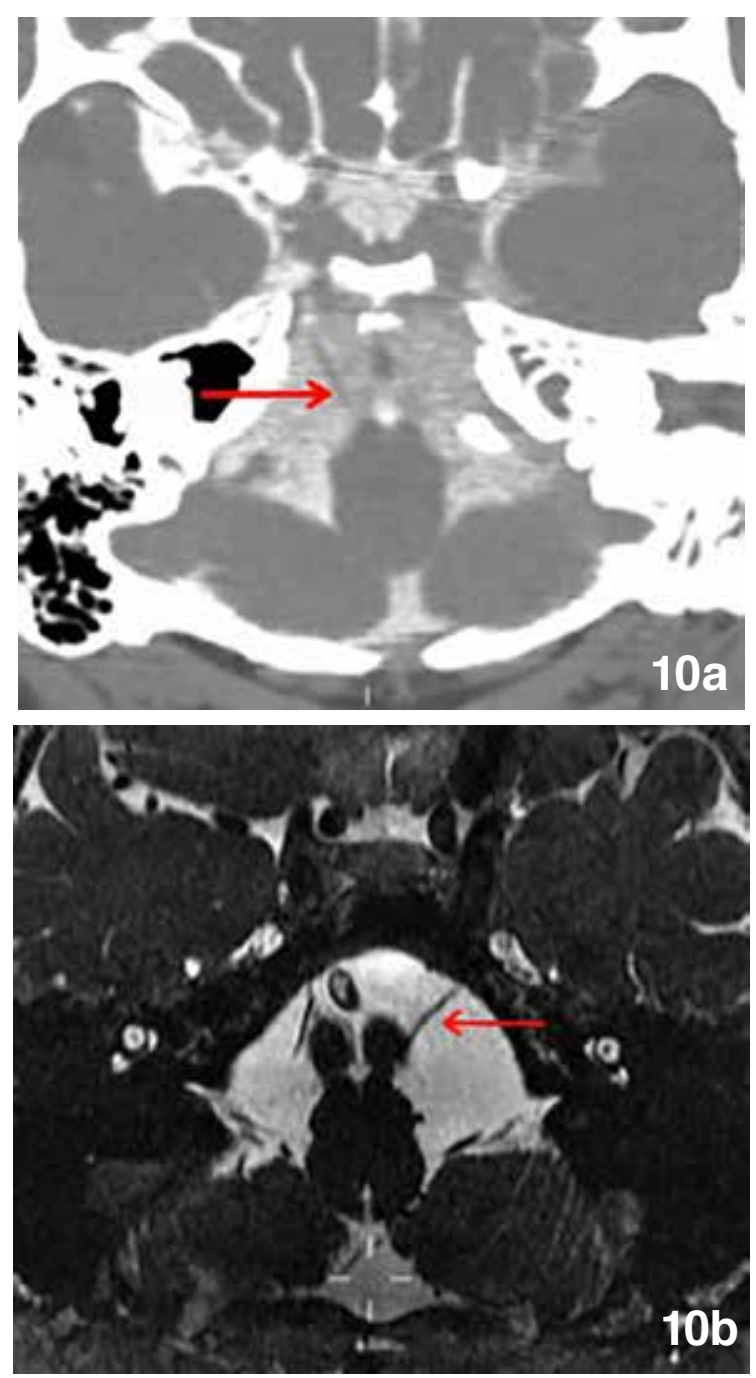

Figura 10: Cortes axiales oblicuos de cisterno TC y RM secuencia FIESTA con visualización del sexto par y canal de Dorello. 


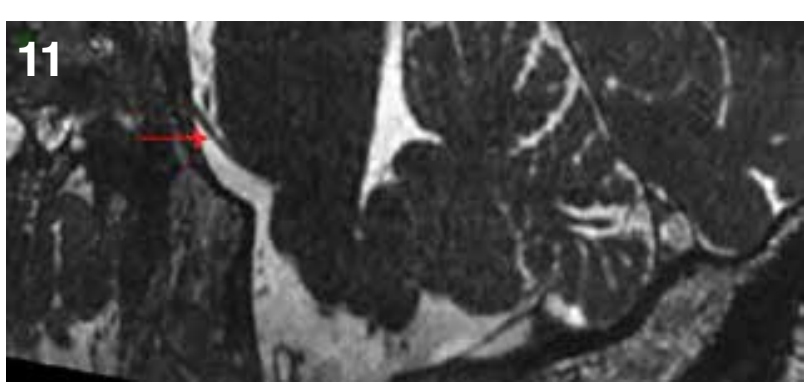

Figura 11: Corte sagital oblicuo de RM en secuencia FIESTA demuestra el trayecto cisternal ascendente del sexto par.

- Segmento del seno cavernoso: Dentro del seno cavernoso, el nervio abducens se localiza inferolateral a la arteria carótida interna, siendo el único nervio que viaja por dentro del seno cavernoso (Figura 6).

- Segmento extraforaminal: Habiendo ingresado a la órbita a través de la fisura orbitaria superior dentro del anillo tendinoso, suministra al recto lateral. Es el nervio más inferior que pasa a través del anillo tendinoso, inferior a la división inferior del nervio motor ocular común ${ }^{(7)}$.

\section{Nervio facial (NC VII)}

Es uno de los nervios craneales clave con una gama compleja y amplia de funciones: nervio motor de la expresión facial que comienza como un tronco y emerge de la glándula parótida como seis ramas, tiene fibras gustativas y parasimpáticas.

El nervio facial es el único nervio craneal que puede mostrar un realce normal después del contraste, aunque esto solo aplica al segmento laberíntico hasta el foramen estilomastoideo, en relación a la presencia de elementos venosos peri y epineurales ${ }^{(8,9,10)}$.

- Segmento nuclear y parenquimatoso: Tres núcleos del tronco cerebral contribuyen al nervio facial; uno motor, uno secretomotor y uno sensorial. Estos tres son: el núcleo del nervio facial en la protuberancia (como las fibras motoras del nervio facial se bifurcan posteriormente sobre el núcleo del nervio abducens, forman el colículo facial en el piso del cuarto ventrículo), el núcleo salival superior (junto al núcleo facial suministra fibras parasimpáticas secretomotoras) y el núcleo del tracto solitario (lateral al núcleo dorsal del nervio vago, suministra fibras gustativas que terminan en la cuerda del tímpano).

- Segmento cisternal: El nervio emerge inmediatamente debajo de la protuberancia, lateral al nervio abducens y medial al nervio vestibulococlear (CN VIII) y se une con el nervio intermedio, que emerge lateral al tronco principal. Juntos, viajan lateralmente a través del ángulo pontocerebeloso al meato acústico interno. El segmento cisternal no da ramas (Figuras 12, 13, 14).

- Segmento canalicular: Habiéndose unido al nervio intermedio, se encuentran en el cuadrante anterosuperior del conducto auditivo interno, por encima de la crista falciformis y por delante de la barra de Bill. El segmento meatal no da ramas (Figuras 12 y 13).

- Segmento laberíntico (conducto auditivo interno al ganglio geniculado): A medida que el nervio facial y el nervio intermedio pasan a través del cuadrante anterio-superior del meato acústico interno, ingresa al acueducto o canal de Falopio, pasando antero-lateralmente entre la cóclea (anterior) y el vestíbulo (posterior)
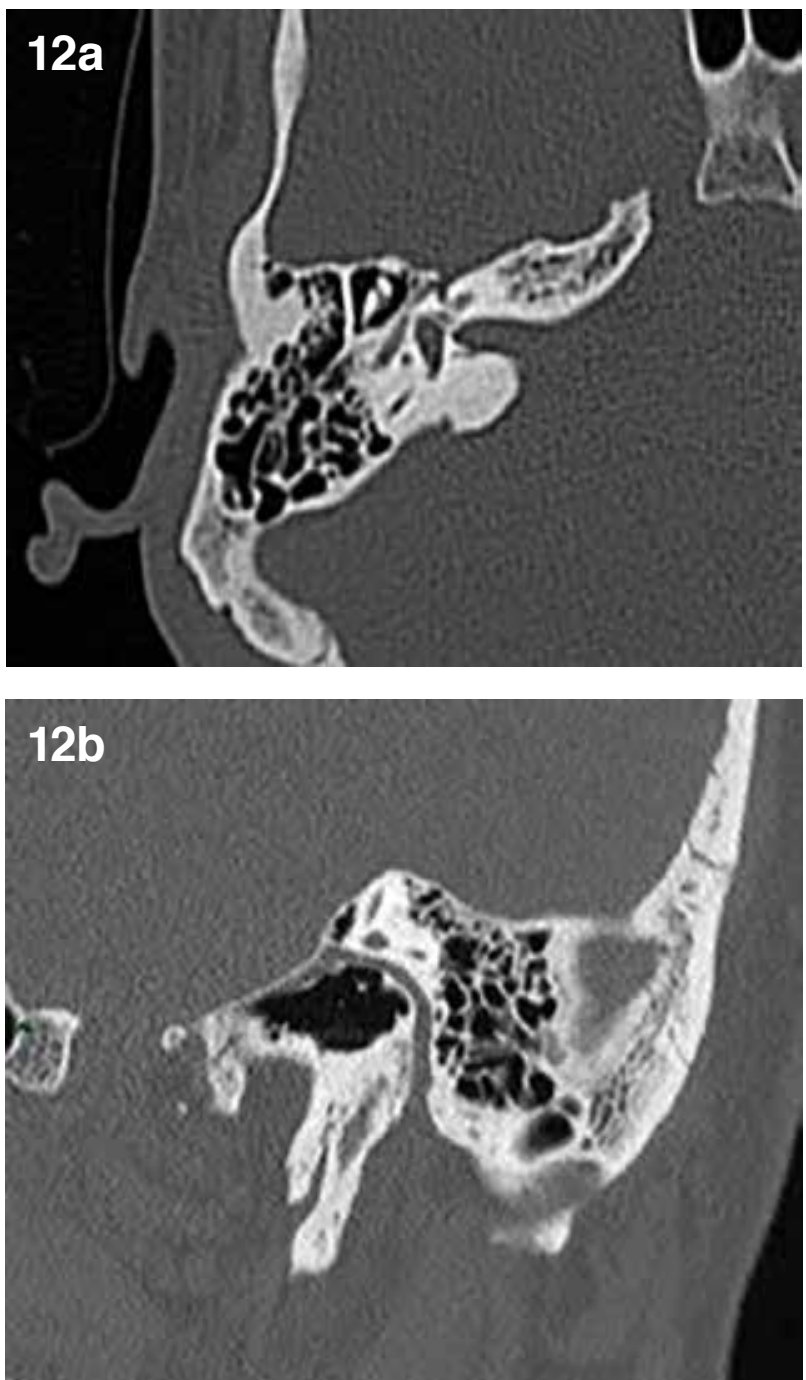

Figura 12: Corte axial y sagital de trayecto laberíntico, ganglio geniculado, trayecto timpánico, segunda rodilla y trayectomastoideo del canal facial. 

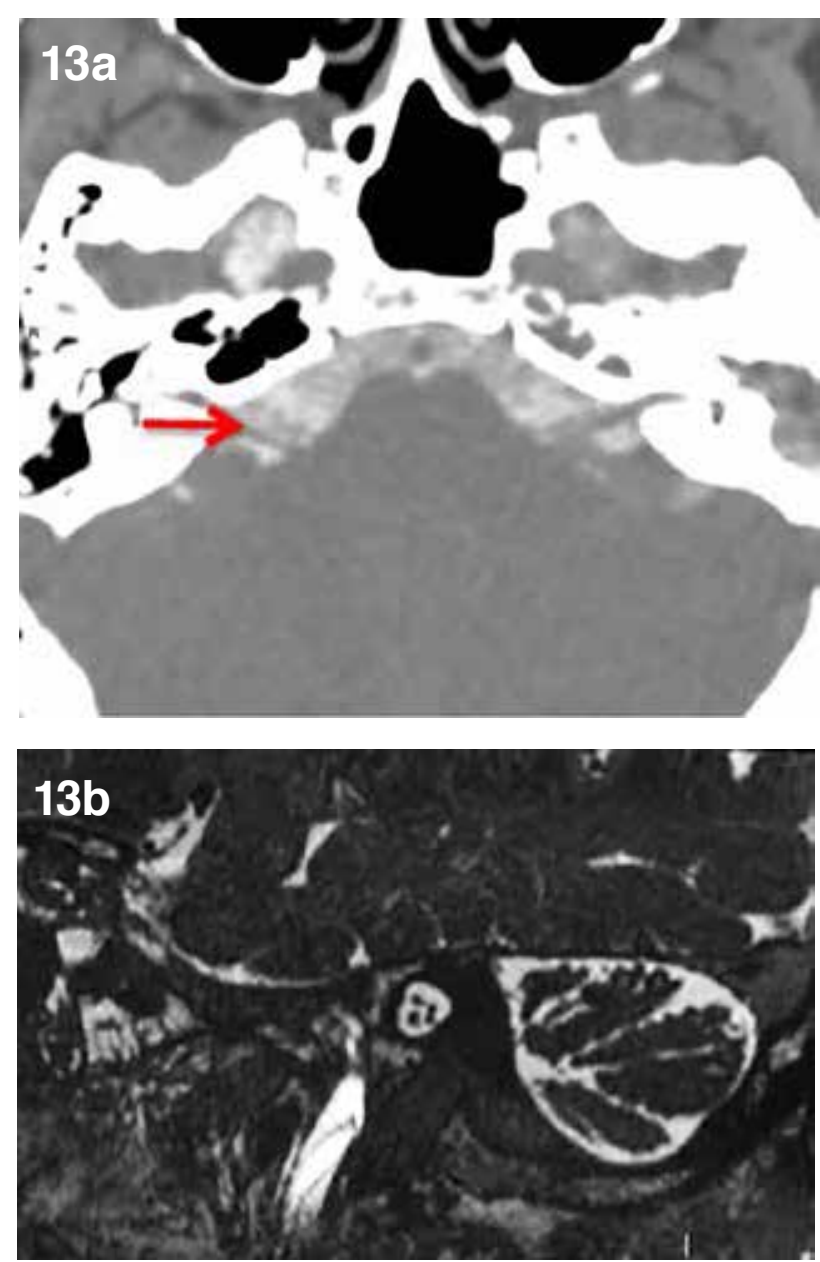

Figura 13: Corte axial en TC en ventana ósea (a) y Cisterno TAC (b) del conducto auditivo interno. (b) Ingreso por el agujero auditivo interno del nervio facial (VII) y vestibulococlear (VIII). Corte sagital oblicuo (c) del CAl en secuencia FIESTA de RM, con los nervios facial (superior y anterior), coclear (inferior y anterior), vestibulares superior e inferior (posteriores).

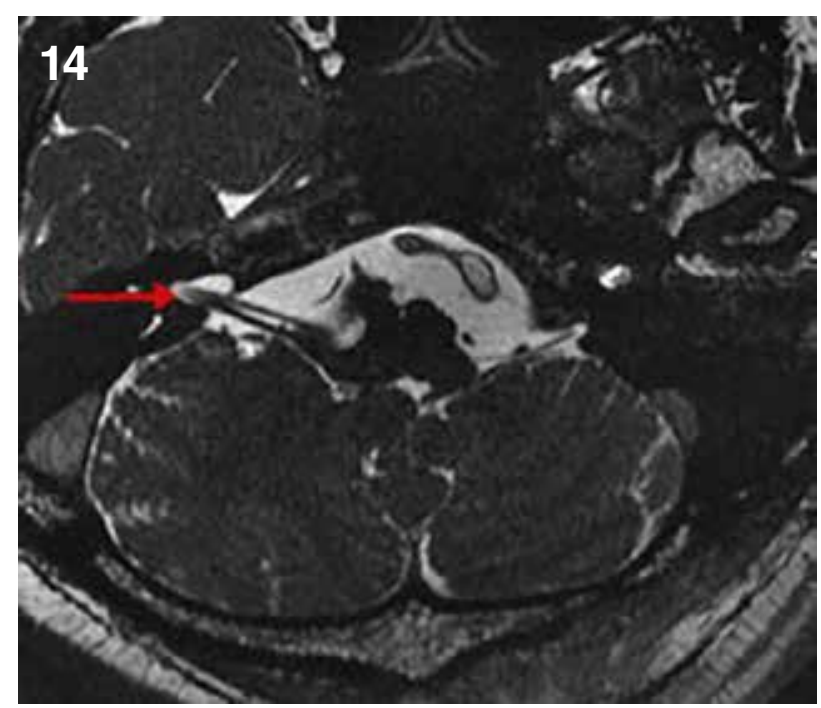

Figura 14: Corte axial oblicuo de RM en secuencia FIESTA, demuestra el VII par derecho y el nervio vestibular superior. y luego dobla hacia posterior en el ganglio geniculado, constituyendo la primera rodilla o genu anterior. Es aquí donde se originan tres ramas: nervio petroso superficial mayor, nervio petroso menor y nervio petroso externo. El segmento laberíntico es el más corto, el más estrecho y el más susceptible al compromiso vascular (Figura 12a).

- Segmento timpánico (desde el ganglio geniculado hasta la eminencia piramidal): A medida que el nervio pasa hacia posterior desde el ganglio geniculado se convierte en el segmento timpánico y se encuentra inmediatamente debajo del canal semicircular lateral en la pared medial del oído medio. El hueso del canal de Falopio a menudo es dehiscente en el área de la ventana oval en el $25-55 \%$ de las muestras post mórtem, teniendo la mucosa en contacto directo con el nervio, susceptible para lesión quirúrgica accidental. El nervio pasa posterior al proceso cocleariforme, tensor timpánico y ventana oval. Apenas distal a la eminencia piramidal, el nervio realiza un segundo giro (rodilla posterior) que se dirige verticalmente hacia inferior formando el segmento mastoideo. El segmento timpánico no da ramas ${ }^{(11)}$ (Figura 12).

- Segmento mastoideo: se extiende desde la parte posterior de la segunda rodilla hacia el agujero estilomastoideo, a través de lo que se conoce como el canal de Falopio. Va por la pared medial del aditus ad antrum de la mastoides. Emite tres ramas: nervio del estapedio, nervio de la cuerda del timpano (rama terminal del nervio intermedio que lleva fibras secretomotoras hacia la glándula submandibular y sublingual y fibras del gusto hacia los dos tercios anteriores de la lengua) y nervio de la rama auricular del nervio vago (fibras de dolor en la parte posterior del conducto auditivo externo y del agujero de la yugular) (Figura 12).

- Segmento extraforaminal: da 9 ramas. A medida que el nervio emerge del foramen estilomastoideo, emite el nervio auricular posterior que inerva parte del meato acústico externo, la membrana timpánica, los músculos auriculares y la parte occipital del occipitofrontal. Luego pasa entre el vientre posterior del músculo digástrico y el músculo estilohioideo, inervando ambos y entra a la glándula parótida. Situado entre los lóbulos superficial y profundo de la glándula, posterior y habitualmente lateral a la vena retromandibular, el nervio se divide en dos ramas principales en el pes anserinus (del latín pata de pato): ramas temporofaciales superiores e inferiores cervicofaciales. Del borde anterior de la glándula, emergen cinco 
ramas; temporal, cigomática, bucal, mandibular o marginal y cervical(7).

\section{Nervios cocleares y vestibulares (NC VIII)}

Es el octavo par craneal y tiene dos funciones: inervación a la cóclea para la audición e inervación al vestíbulo para el equilibrio.

- El nervio coclear emerge de los núcleos coclear posterior y anterior, en cambio, el nervio vestibular emerge de los núcleos de la zona vestibular del piso del IV ventrículo.

- Emergen entre la protuberancia y la médula, lateral al nervio facial y al nervio intermedio, pasando lateralmente a través del ángulo pontocerebeloso al meato acústico interno.

- En el meato acústico interno, el nervio se divide en 4 haces: nervio coclear, división superior e inferior del nervio vestibular y rama de este último, el nervio del canal semicircular posterior (o nervio singular).

- Nervio coclear: Se transmite con las células sensoriales en el ganglio espiral, que está en la base de la lámina espiral de la cóclea (Figuras 13 y 15).

- Nervio vestibular: Del nervio vestibular en el ganglio vestibular o de Scarpa, surgen tres haces:

- La división superior del nervio vestibular transporta fibras sensoriales de las células ciliadas de los conductos semicirculares anterior y lateral y del utrículo. Está ubicado en el cuadrante posterosuperior (Figuras 13 y 14).

- La división inferior transporta fibras sen-

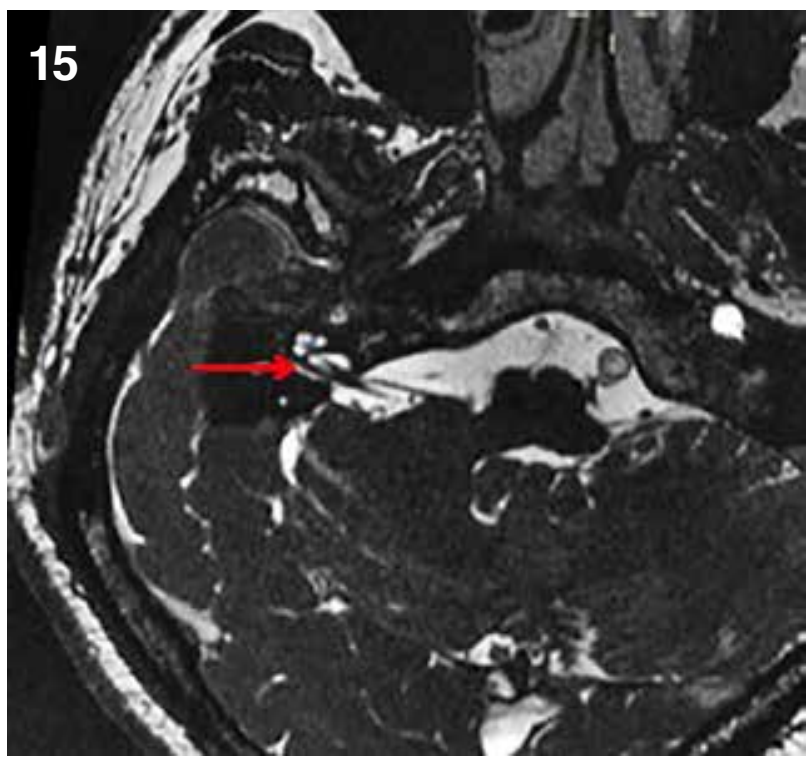

Figura 15: Corte axial oblicuo de RM secuencia FIESTA demuestra nervios cocleares y vestibular inferior además de cóclea derecha. soriales del sáculo. Está ubicado en el cuadrante posteroinferior (Figuras 13 y 15).

- El nervio singular, también ubicado en el cuadrante posteroinferior, pasa a través del foramen singular ${ }^{(7)}$.

\section{Pares bajos}

A continuación, se detalla solamente el trayecto cisternal y foraminal del IX, X, XI y XII pares craneales, debido a su difícil representación por imágenes del trayecto cisternal y en el cuello.

Nervio Glosofaríngeo (NC IX): emerge del bulbo raquídeo desde el surco postolivar, luego pasa lateralmente al flóculo y abandona el cráneo a través de la pars nervosa del foramen yugular en una vaina separada de la duramadre ${ }^{(7)}$.

Nervio Vago (NC X): las fibras de los núcleos centrales emergen de la médula en el surco postolivar, entre el nervio glosofaríngeo (NC IX) y la raíz craneal del nervio accesorio (NC XI). Desde aquí, el nervio vago viaja a través de la cisterna magna para emerger del cráneo a través de la pars vascularis del foramen yugular para ingresar al espacio carotídeo ${ }^{(7)}$.

Nervio accesorio o espinal (NC XI): Se compone de dos partes, la parte craneal y la parte espinal (Figura 16). La parte craneal (segmento accesorio) es la más pequeña de las dos. Las fibras de la parte espinal (segmento espinal) surgen de las células del cuerno ventral en el cordón entre $\mathrm{C} 1$ y $\mathrm{C} 5$ del plexo cervical. Las fibras emergen del cordón lateralmente entre las raíces nerviosas espinales anterior y posterior para formar un solo tronco, que asciende al cráneo a través del foramen magno. Emerge del cráneo a través del foramen yugular ${ }^{(7)}$.

Nervio Hipogloso (NC XII): Proporciona a la lengua su control motor (Figura 16) El nervio emerge

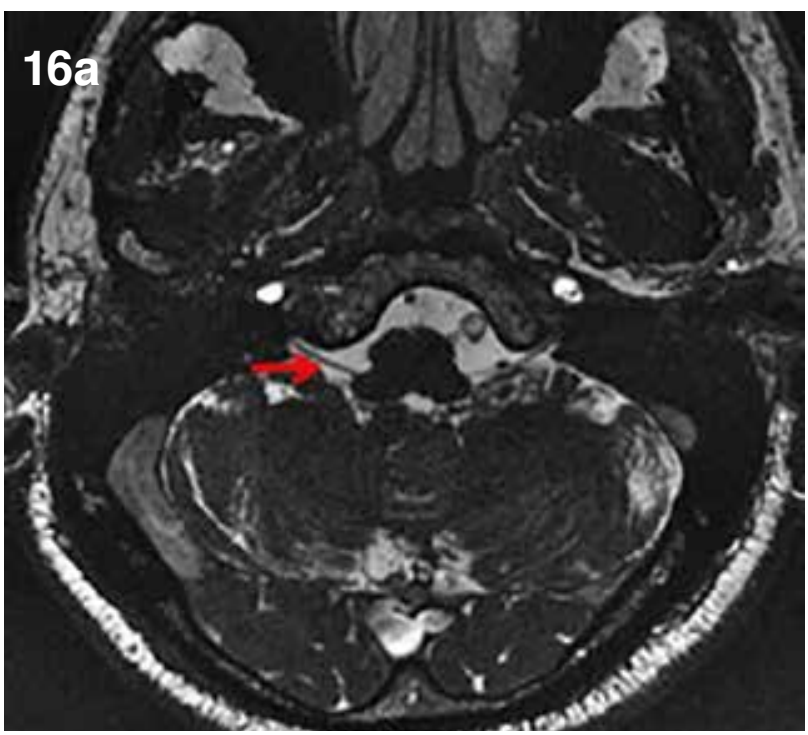




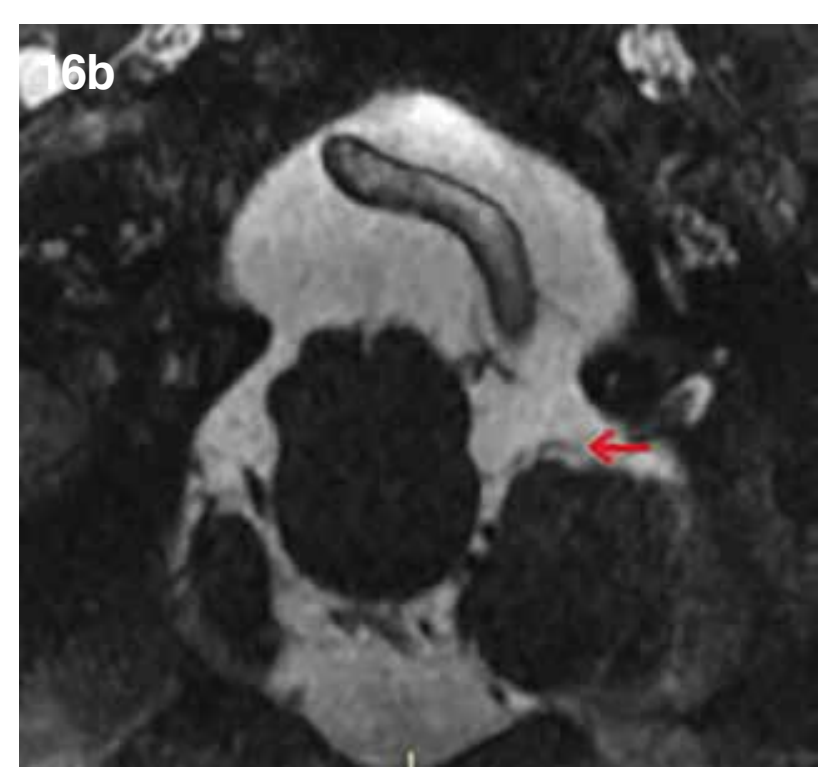

Figura 16: Cortes axiales de RM secuencia FIESTA, demuestra el trayecto del nervio hipogloso (a) y más caudal en los pares bajos (b).

del bulbo lateralmente. Estas fibras se unen para formar dos raíces, y pasando por anterior, ingresan al canal hipogloso y emerge por inferior de la base del cráneo. Dentro del canal, las dos raíces se unen, de modo que surge un único nervio ${ }^{(7)}$.

\section{Conclusión}

La base del cráneo forma el piso de la cavidad craneal que separa el cerebro de las estructuras faciales y el cuello suprahioideo. La anatomía de la base del cráneo es compleja y no está directamente accesible para la evaluación clínica. En la base del cráneo se pueden identificar tres regiones naturalmente contorneadas cuando se ve desde superior, las fosas craneales anterior, media y posterior. Se observan numerosos foramen y canales en la base del cráneo, que transmiten estructuras neurovasculares vitales. La TC y la RM son la modalidad de imagen de elección para la evaluación de la anatomía y la patología de la base del cráneo y cumplen un rol complementario. El trayecto de los nervios craneales sigue un recorrido conocido y su segmentación permite el adecuado estudio y visualización con estas técnicas.

\section{Referencias}

1. Sheth S, Branstetter BF, Escott EJ. Appearance of normal cranial nerves on steady-state free precession MR images. Radiographics. 2009 jul; 29(4): 1045-1055.

2. Valenzuela R, Ebensperguer E. Base de cráneo: Anatomía y patología tumoral. Revisión conceptual. Rev Chil Radiol. 2002; 8(4): 170-176.

3. Miranda A, Kuschel C, Miranda M, Fuentes A. Anatomía radiológica de la base de cráneo y los nervios craneales parte 1: Generalidades y base de cráneo. Rev Chil Radiol. 2018 jul; 24(3): 105-111.

4. Martín Al, Pertusa E, Cajal B, Dominguez E, Galvan R, Molina JF. Radiologic Anatomy of the cranial nerves. Poster presented at the European Congress of Radiology. Madri, España. European Society of Radiology. 2013.

5. Casselman J, Mermuys K, Delanote J, Ghekiere J, Coenegrachts K. MRI of the cranial nerves-more than meets the eye: technical considerations and advanced anatomy. Neuroimaging Clin N Am. 2008 may 1; 18(2): 197-231.

6. Blitz AM, Choudhri AF, Chonka ZD, Ilica AT, Macedo LL, Chhabra A, Gallia GL, Aygun N. Anatomic considerations, nomenclature, and advanced cross-sectional imaging techniques for visualization of the cranial nerve segments by MR imaging. Neuroimaging Clin N Am. 2014 feb 1; 24(1): 1-5.

7. Foster T, Gaillard F. Radiopaedia.org: Cranial nerves. Available at: https://radiopaedia.org/articles/cranialnerves. Accessed June 4, 2020.

8. Thurston M, Gaillard F. Radiopaedia.org: Normal facial nerve enhancement on MRI. Available at: https://radiopaedia.org/articles/normal-facial-nerve-enhancementon-mri. Accessed June 4, 2020.

9. Radhakrishnan R, Ahmed S, Tilden JC, Morales H. Comparison of normal facial nerve enhancement at 3T MRI using gadobutrol and gadopentetate dimeglumine. Neuroradiol J. 2017 Dec; 30(6): 554-560.

10. Saremi F, Helmy M, Farzin S, Zee CS, Go JL. MRI of cranial nerve enhancement. Am J Roentgenol. 2005 dec; 185(6): 1487-1497.

11. Yetiser $S$. The dehiscent facial nerve canal. International Journal of Otolaryngology. 2012 Feb 21; 2012(6): 679708 